\title{
Barrières relatives aux changements en pharmacie : perspective d'étudiants canadiens en pharmacie
}

La pratique pharmaceutique évolue $e^{1-4}$. Afin de guider son évolution, plusieurs sociétés savantes proposent leur vision de la pratique pharmaceutique assortie de plans directeurs inspirés des meilleures données probantes et des besoins de la population ${ }^{5-8}$. Ces plans directeurs influencent également le cadre normatif proposé par les organismes d'agrément, les ordres professionnels et les facultés de pharmacie?.

En dépit de ces balises, force est de constater la présence de retards importants entre l'émergence de ces normes et leur implantation à large échelle. Des enquêtes européennes, américaines et canadiennes montrent la lente adoption (c.-̀̀-d. plusieurs décennies) des meilleures pratiques pharmaceutiques (p. ex. distribution unitaire quotidienne, décentralisation des pharmaciens dans les programmes de soins, réalisation de bilans 
Tableau 1. Perception des barrières relatives au changement par 225 étudiants canadiens en pharmacie

Barrières relatives au changement*

Absence de communication efficace tout au long de

l'implantation

Absence de leadership

Absence de collaboration

Absence de plan de match clair ou de vision stratégique

Absence de culture favorable au changement

Absence de compréhension de ce qui est à faire par

les personnes visées par le changement

Absence de ressources financières

Absence de vision commune

Absence de sentiment d'urgence

Absence d'appuis formels de l'équipe de gestion

qui fait du changement une vraie priorité

Absence de formation adéquate des personnes visées

spécifique au changement à implanter

Absence de prise en compte des antécédents d'échecs

au changement

Absence de confiance

Absence de description optimale des tâches aux

personnes visées par le changement

Absence d'appuis stratégiques au sein de l'hôpital

Absence de retombées favorables pour le patient

Absence de consultation préalable et adéquate des

personnes visées par le changement

Absence d'anticipation des effets réels du changement ....

Absence de climat de travail propice

Absence de réajustements/corrections apportés aux

processus en cours d'implantation

Absence de prise en compte de la résistance exprimée

par certaines personnes visées par le changement

Absence d'identification des obstacles potentiels au

changement

Absence de ressources humaines

Absence de marqueurs de résultats du changement

faciles à suivre

Absence de ressources matérielles

Absence de prise en compte des inquiétudes exprimées

par les personnes visées par le changement

Absence d'autonomie suffisante donnée à ceux visés par le changement

Absence de formation générale sur la gestion du changement

Absence de rétroaction/soutien/encouragement de la part de l'équipe de gestion

\begin{tabular}{ccc}
$\begin{array}{c}\text { Somme des } \\
\text { cotationst }\end{array}$ & $\begin{array}{c}\text { Moyenne de la } \\
\text { cotation‡ }\end{array}$ & $\begin{array}{c}\text { Somme des } \\
\text { rangs attribués§ }\end{array}$ \\
\hline 361 & 1,6 & 242
\end{tabular}

Facteurs selon

Parkin $^{18}$

Climat et

communication

Leadership et

communication

Climat et

communication

Buts, priorités

et performance

Structure,

stratégie et culture

Organisation,

connaissance

et information

Ressources

Buts, priorités et performance

Buts, priorités et performance

Buts, priorités et performance

Organisation, connaissance et information

Structure, stratégie et culture

Climat et communication

Organisation, connaissance et information

Buts, priorités et performance

Organisation, connaissance et information

Structure, stratégie et culture Buts, priorités et performance

Climat et communication

$4842,2 \quad 113 \quad \begin{gathered}\text { Climat et } \\ \text { communication }\end{gathered}$

$\begin{array}{lll}2,2 & 63 & \begin{array}{c}\text { Processus } \\ \text { interpersonnel }\end{array}\end{array}$

$\begin{array}{llll}490 & 2,2 & 59 & \text { Processus }\end{array}$
interpersonnel

$\begin{array}{llll}496 & 2,2 & 94 & \text { Structure, }\end{array}$
stratégie et culture Ressources

Buts, priorités et performance

Ressources

$\begin{array}{llll}513 & 2,3 & 52 & \text { Ressources } \\ 515 & 2,3 & 46 & \text { Processus }\end{array}$
interpersonnel

Organisation, connaissance et information

Organisation, connaissance et information

Processus interpersonnel

Suite à la page 200 


\section{Tableau 1. Perception des barrières relatives au changement par 225 étudiants canadiens en pharmacie (suite et fin)}

\begin{tabular}{|c|c|c|c|c|}
\hline Barrières relatives au changement* & $\begin{array}{c}\text { Somme des } \\
\text { cotationst }\end{array}$ & $\begin{array}{c}\text { Moyenne de la } \\
\text { cotation‡ }\end{array}$ & $\begin{array}{c}\text { Somme des } \\
\text { rangs attribués§ }\end{array}$ & $\begin{array}{l}\text { Facteurs selon } \\
\text { Parkin }^{18}\end{array}$ \\
\hline $\begin{array}{l}\text { Absence de reconnaissance des personnes visées par } \\
\text { le changement }\end{array}$ & 537 & 2,4 & 18 & $\begin{array}{l}\text { Processus } \\
\text { interpersonnel }\end{array}$ \\
\hline $\begin{array}{l}\text { Absence de fondement scientifique ou de données } \\
\text { probantes justifiant le changement }\end{array}$ & 538 & 2,4 & 113 & $\begin{array}{l}\text { Organisation, } \\
\text { connaissance et } \\
\text { information }\end{array}$ \\
\hline $\begin{array}{l}\text { Absence de mise en place de personnes responsables } \\
\text { de l'instauration des changements }\end{array}$ & 539 & 2,4 & 17 & $\begin{array}{l}\text { Structure, } \\
\text { stratégie et culture }\end{array}$ \\
\hline $\begin{array}{l}\text { Absence d'identification des personnes susceptibles } \\
\text { de nuire au changement }\end{array}$ & 565 & 2,5 & 17 & $\begin{array}{l}\text { Structure, } \\
\text { stratégie et culture }\end{array}$ \\
\hline $\begin{array}{l}\text { Absence d'expertise scientifique/technique au sein } \\
\text { de l'équipe }\end{array}$ & 575 & 2,6 & 31 & $\begin{array}{l}\text { Organisation, } \\
\text { connaissance et } \\
\text { information }\end{array}$ \\
\hline Absence de légitimité des personnes concernées & 595 & 2,6 & 12 & $\begin{array}{l}\text { Processus } \\
\text { interpersonnel }\end{array}$ \\
\hline
\end{tabular}

*Par ordre d'importance selon la somme des cotations.

†La somme des cotations est la somme de la cotation attribuée pour chaque facteur (c.-à-d. 1 = très important, 2 = important, 3 = peu important, 4 = pas du tout important) pour les 225 étudiants.

fLa moyenne de la cotation est la somme des cotations divisée par le nombre d'étudiants, à savoir 225.

$\S$ ¿a somme des rangs attribués est la somme des 5 premiers facteurs attribués par les étudiants. Un facteur classé au 1 er rang comptait pour 5 points; un facteur classé au $2^{\mathrm{e}}$ rang, pour 4 points; un facteur classé au 3e rang, pour 3 points; un facteur classé au $4^{\mathrm{e}}$ rang, pour 2 points; un facteur classé au $5^{\mathrm{e}}$ rang, pour 1 point. Les cellules grisées correspondent aux 10 facteurs classés dans les cinq premiers rangs par les étudiants.

comparatifs de médicaments, surveillance environnementale des médicaments dangereux $)^{10-14}$. Après avoir procédé à une revue de la littérature et à l'examen de neuf procédures cliniques applicables aux soins de santé, Balas et Boren ont conclu qu'il fallait environ une quinzaine d'années pour que les meilleures données probantes issues de la recherche soient largement implantées en clinique ${ }^{15}$. Cet article pivot met en évidence les retards importants qui existent entre la publication de données probantes et leur implantation. Depuis la parution de cet article, de nombreux articles ont été publiés sur les concepts de knowledge transfer et de knowledge translation. ${ }^{16}$

Plusieurs facteurs contribuent à la lente adoption des meilleures pratiques. Nous avons recensé 35 barrières au changement pouvant expliquer ces lenteurs d'implantation en pharmacie. Nous avons développé un questionnaire bilingue (français et anglais) d'évaluation de l'importance des barrières au changement en pratique pharmaceutique. Ce questionnaire a été testé auprès de quelques membres de l'unité de recherche et auprès d'un petit groupe d'étudiants en pharmacie. Dans le cadre d'une présentation orale de 60 minutes effectuée lors de la Semaine de développement professionnel des étudiants canadiens en pharmacie tenue à Montréal en janvier $2013^{17}$, nous avons décrit plusieurs pratiques pharmaceutiques fondées sur les meilleures preuves et discuté des retards d'implantation. Les étudiants présents étaient inscrits au programme de pharmacie de la $1^{\mathrm{re}}$ à la $4^{\mathrm{e}}$ année. Après la présentation, le questionnaire papier a été administré à tous les étudiants en pharmacie y ayant assisté. Chaque étudiant était invité à coter l'importance relative de chaque barrière au changement (c.-à-d. très important, important, peu important, pas du tout important) et de hiérarchiser les cinq barrières les plus importantes selon eux, en leur attribuant un rang de 1 à 5. Au total, 225 étudiants ont rempli le questionnaire. Le tableau 1 présente les résultats d'évaluation de l'importance des barrières au changement en pratique pharmaceutique en fonction des facteurs paralysants élaborés par Parkin ${ }^{18}$. Sans surprise, le manque de communication, de leadership, de collaboration, de plan de match clair et de culture favorable au changement ont dominé le classement des facteurs paralysants. Fait étonnant, l'absence d'éléments objectifs, tels les marqueurs de résultats du changement et les fondements scientifiques ou les preuves justifiant le changement ont beaucoup moins pesé dans la balance. Notons enfin qu'il existait une cohérence entre le classement des facteurs et les cinq facteurs jugés les plus importants par les étudiants.

Les étudiants en pharmacie représentent la génération de demain et ils seront plus que jamais interpellés par le changement. Cette présentation orale a sensibilisé les étudiants en pharmacie aux barrières au changement. Les cursus universitaires devraient intégrer ces notions relatives au changement et à l'évolution des pratiques pharmaceutiques. Les organismes réglementaires et les ordres professionnels devraient également contribuer à faire de la gestion du changement une priorité, en soutenant les professionnels dans ce type de démarche et en les outillant davantage. Reconnaitre les barrières au changement peut aider à les faire tomber, à les éviter ou à les prévenir !

\section{Références}

1. Pearson GJ. Evolution in the practice of pharmacy—not a revolution! $C M A J$. 2007:176(9):1295-6.

2. Holland RW, Nimmo CM. Transitions, part 1: beyond pharmaceutical care. Am J Health Syst Pharm. 1999;56(17):1758-64.

3. Abramowitz PW. The evolution and metamorphosis of the pharmacy practice model. Am J Health Syst Pharm. 2009;66(16):1437-46.

4. Abramowitz PW, Shane R, Daigle LA, Noonan KA, Letendre DE. Pharmacist interdependent prescribing: a new model for optimizing patient outcomes. Am J Health Syst Pharm. 2012;69(22):1976-81.

5. 2020 vision - FIP's vision, mission and strategic plan. La Hague (Pays Bas) : Fédération internationale de pharmacie. Publié au: https://www.fip.org/ 
files/fip/strategic\%20plan\%20no\%20annexes.pdf. Consulté le 31 janvier 2013.

6. 2015 initiative. Bethesda (MD) : American Society of Health-System Pharmacists. Publié au : www.ashp.org/2015. Consulté le 31 janvier 2013.

7. Blueprint for pharmacy. Ottawa (ON) : Association des pharmaciens du Canada. Publié au: www.pharmacists.ca/index.cfm/pharmacy-incanada/blueprint-for-pharmacy/. Consulté le 31 janvier 2013.

8. CSHP 2015-targeting excellence in pharmacy practice. Ottawa $(\mathrm{ON})$ : Société canadienne des pharmaciens d'hôpitaux; 2013. Publié au : www.cshp.cal programs/cshp2015/index_e.asp. Consulté le 31 janvier 2013.

9. Normes de gestion des médicaments. Ottawa $(\mathrm{ON})$ : Agrément Canada. Publié au : www.accreditation.ca/fr/content.aspx?pageid=285\&rdr=true\&LangType $=3084$. Consulté le 31 janvier 2013 .

10. EAHP's 2010 survey of hospital pharmacy practice in Europe. Bruxelles (Belgique) : European Association of Hospital Pharmacists; 2013. Publié au : www.eahp.eu/publications/survey/eahps-2010-survey-hospital-pharmacypractice-europe. Consulté le 31 janvier 2013.

11. Pedersen CA, Schneider PJ, Scheckelhoff DJ. ASHP national survey of pharmacy practice in hospital settings: prescribing and transcribing-2010. Am J Health Syst Pharm. 2011;68(8):669-88.

12. Pedersen CA, Schneider PJ, Scheckelhoff DJ. ASHP national survey of pharmacy practice in hospital settings: dispensing and administration-2011. Am J Health Syst Pharm. 2012;69(9):768-85.

13. Pedersen CA, Schneider PJ, Scheckelhoff DJ. ASHP national survey of pharmacy practice in hospital settings: monitoring and patient education2009. Am J Health Syst Pharm. 2010;67(7):542-58.

14. Babich M, Bornstein, C, Bussières JF, Hall K, Harding J, Lefebvre P, et al., rédacteurs. Rapport annuel 2009-2010 sur les pharmacies hospitalières canadiennes. Publié au : www.lillyhospitalsurvey.ca/hpc2/content/rep_2010_tocF.asp. Consulté le 31 janvier 2013.

15. Balas EA, Boren SA. Managing clinical knowledge for health care improvement. Yearb Med Inform. 2000;(1):65-70.

16. Curran JA, Grimshaw JM, Hayden JA, Campbell B. Knowledge translation research: the science of moving research into policy and practice. J Contin Educ Health Prof. 2011;31(3):174-80.

17. Bussières JF. Le boson de Bussières ou les enjeux clés de la pratique pharmaceutique hospitalière [présentation]. Semaine de développement professionnel de l'Association canadienne des étudiants et internes en pharmacie; du 9 au 12 janvier 2013; Montréal (Québec). Publié au : http://urppchusj.wordpress.com/2013/01/12/pdw2013/ . Consulté le 31 janvier 2013.

18. Parkin P. Managing change in healthcare using action research. London (Angleterre) : Sage Publications Ltd; 2009.

\section{Aurélie Guérin}

Assistante de recherche

Jean Rémi Valiquette

Assistant de recherche

Denis Lebel, B. Pharm., M. Sc., FCSHP

Adjoint

Jean-François Bussières, B. Pharm., M. Sc., FCSHP

Chef

Unité de recherche en pratique pharmaceutique

Département de pharmacie

Centre hospitalier universitaire Sainte-Justine

Montréal, Québec

Aurélie Guérin est aussi candidate au D. Pharm. à I'Université Paris Sud XI, Paris, France. Jean Rémi Valiquette est aussi candidat pour le degré PharmD à l'Université de Montréal. Jean-François Bussières est aussi professeur, Faculté de pharmacie, Université de Montréal, Montréal (Québec).

Divulgation d'intérêts concurrents : Aucun déclarés. 